\title{
A traditional demonstration of the active properties of Pavlovian inhibition using differential CER
}

LYNN J. HAMMOND

TEMPLE UNIVERSITY

Rats in an experimental group were given 30 trials of differential CER and then the $\mathrm{CS}^{+}$and $\mathrm{CS}$ - were combined during CER extinction. The combination resulted in less suppression for the experimental group than shown by a control group which had a $\mathrm{CS}^{+}$and a formerly random stimulus combined during extinction. This was interpreted as a demonstration of the active inhibitory properties of $C S-$.

From its inception, Pavlovian conditioning has been said to consist of two active processes: Stimuli associated with reinforcement acquire excitatory properties, while stimuli associated with the absence of reinforcement acquire inhibitory properties. In many of the instances in which inhibition is hypothesized, the effect to be explained consists of an absence of the CR. Often one could just as well attribute such an outcome to the absence of excitation and maintain a more parsimonious explanation. Pavlov, however, made it quite clear that he viewed all inhibitory effects as the result of an active process. When this question first arose in his lectures in 1924, he stated that "The action of the additional stimulus (in this case a conditioned inhibitor) can be tested, however, by applying it in combination with some other positive conditioned stimulus with which it has never been associated" (Pavlov, 1960, p. 75). He then described several experiments by Leporsky and Babkin in which well developed excitatory and inhibitory stimuli were presented together for the first time and a markedly diminished $C R$ was observed compared to that found for the excitatory stimulus alone. This type of demonstration was also shown with humans (Rodnick, 1937) using an inhibitory stimulus elaborated during GSR conditioning and an excitatory stimulus from eyelid conditioning.

The present experiment was done within the context of contemporary two-process learning theory (Rescorla \& Solomon, 1967). In this approach, stimuli from Pavlovian conditioning are observed to interact with instrumental responding. It has been demonstrated in such interaction studies that stimuli conditioned by Pavlovian procedures can exercise strong control over instrumental response rate. One of the major tenets of this two-process approach is that Pavlovian inhibitory stimuli will produce motivational effects opposite to those produced by excitatory stimuli. Rescorla and Solomon, like Pavlov, conceptualize inhibition as an active process. This is easily demonstrated in many interaction studies, because the response measure, change in instrumental rate, is bidirectional and, therefore, a CS- can produce a rate change in the opposite direction from that of a CS+ (e.g., Rescorla \& LoLordo, 1965).

In the CER situation where shock conditioning is superimposed upon appetitively motivated instrumental responding, a differential CS- or "safety" signal has produced facilitation of bar press responding (Hammond, 1966). However, this facilitation was only transitory; but one can argue that although a CS- may be an active inhibitor of fear, no facilitation will occur unless fear is present to be inhibited. Thus the question still remains as to whether a CS- has active inhibitory properties in the later stages of differential CER.

In the present experiment, this question was examined in a manner similar to the older Pavlovian experiments of Leporsky and Babkin. After a period of differential CER procedure, the CS+ and CS- were presented together during Pavlovian extinction. If the CS- is an active inhibitor of fear it should at least diminish the suppression produced by the conditioned fear stimulus. However, any stimulus combined with a CS+ could result in a diminished $\mathrm{CR}$ because of stimulus generalization decrement. Consequently, a control was included which utilized the random control procedure during the conditioning phase of the experiment. In this procedure a stimulus occurs in random relationship with the US (Rescorla, 1967). It is the ideal control procedure for the measurement of inhibition and represents a considerable refinement of the original salivation experiments done in Pavlov's laboratory.

Method

Twenty albino rats were divided equally into an inhibition group (I) and a random control group (R).

Pretraining, Days 1-6: Ten automatically programmed Skinner boxes were used. Ss were shaped to bar press for water reward (under $21-1 / 2 \mathrm{~h}$ water deprivation) and then given daily $2 \mathrm{~h}$ sessions of VI-1 min training for the remainder of the experiment. On both the fifth and sixth day of VI training, a flashing light (6 W bulb, .5 sec on-.5 sec off cycle) and a tone (3000 cps, moderate intensity) were each presented three times with random ITI and order. Stimulus duration was always 3 min. No shock was presented on these days.

Stage 1, Days 7-16: During the next 10 session the daily tone (CS+) trials always terminated in a $.5 \mathrm{sec}$, $.72 \mathrm{~mA}$, scrambled grid shock for all Ss. Both groups 


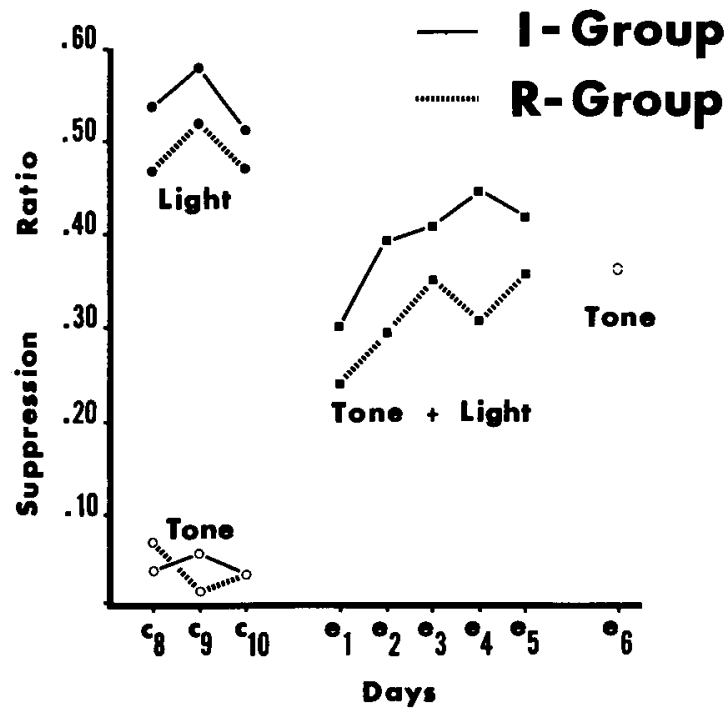

Fig. 1. Daily suppression ratios for the experimental and control groups for later days of the experiment. "c"=conditioning (Stage 1), "e"=extinction during Stage $2\left(e_{1}+e_{5}\right)$ and during Stage $3\left(e_{6}\right)$.

received 30 tone conditioning trials during the 10 days of this stage, but they differed in the relationship of their 30 light trials to the tone-shock trial units. The I group received differential CER: The three daily light (CS-) trials never occurred during a tone-shock trial or during the $3 \mathrm{~min}$ period preceding each toneshock trial. In the R group, on the other hand, the light stimulus was presented three times each day on a random schedule by a separate tape programmer for each S. Since an additional tape programmer presented the three daily tone-shock trials on a random schedule for all Ss, the light occurred randomly and independently of the tone-shock trials for the $R$ group during this stage. (In contrast, the light in the I group was explicitly unpaired with the tone-shock trials.)

Stage 2, Days 17-21: Shock was discontinued and both groups were treated identically. The effects of the light stimuli upon the extinction of conditioned fear were tested by presenting the light and tone simultaneously for $3 \mathrm{~min}$, three trials dally, for the next five days.

Stage 3, Day 22: On the last day of the experiment, neither light nor shock occurred and three tone-alone trials were presented.
Daily suppression ratios for each $S$ were calculated for response to the stimulus or stimulus compound on each day $(A / A+B$, where $A$ is the sum of the rates during the $3 \mathrm{~min}$ stimulus and $B$ is the sum of the rates during the $3 \mathrm{~min}$ period immediately preceding the stimulus).

\section{Results and Discussion}

Dally suppression ratios to the stimuli for both groups appear in Fig. 1. Both groups show almost complete suppression to the tone (CS+) but no suppression to the light stimuli during the last three days of Stage 1.

During extinction (Stage 2), combining the light stimulus with the CS+ produced a significant, differential effect upon the amount of suppression for the two groups $(F=4.77, d f=1 / 90, p<.05)$. There were no significant differences between the days of Stage 2 and, therefore, no course of extinction was observed.

When the light stimulus was removed and tone (CS+) tested alone (Stage 3), suppression remained the same for the R group, but increased (not significantly) for the I group.

It would appear that the difference between groups during Stage 2 is the result of active inhibitory properties of CS- in the I group which act antagonistically to the excitatory properties of CSt. It can also be concluded that the CS- in differential CER has acquired lasting inhibitory properties whlch are still present after later stages of acquisition (i.e., after 30 differential trials).

\section{References}

HAMMOND, L. J. Increased responding to CS- in differential CER. Psychon. Sci, 1966, 5, 337-338.

PAVLOV, I. P. Conditioned reflexes. New York: Dover Publication, 1960.

RESCORLA, R. A. Pavlovian conditioning and its proper control procedures. Psychol Rev., 1967, 74, 71-80.

RESCORLA, R. A., \& LoLORDO, V. M. Inhibition of avoidance behavior. J. comp. physiol Psychol, 1965, 59, 406-412.

RESCORLA, R. A., \& SOLOMON, R. L. Two-process leaming theory: Relationships between Pavlovian conditioning and instrumental learning. Psychol, Rev., 1967, 74, 151-182.

RODNICK, E. H. Does the interval of delay of conditioned responses possess inhibitory properties? J. exp. Psychol, 1937, 20, 507-527. Note

1. This research was supported by NIH grant No. MH-13298-1 and a grant from Temple University. The author thanks Dr. Ronald Baenninger for helpful criticism of the manuscript. 